\title{
Nach der pharmakologischen Euphorie - Eine neue Chance für die Entwicklung und Umsetzung psycho- sozialer Interventionen
}

\author{
After the Pharmacology-Driven Euphoria - A new Chance for Developing \\ and Implementing of Psychosocial Interventions
}

Autoren

Institute
Dirk Richter ${ }^{1}$, Holger Hoffmann ${ }^{2}$

Fachbereich Gesundheit, Berner Fachhochschule

2 Universitäre Psychiatrische Dienste Bern

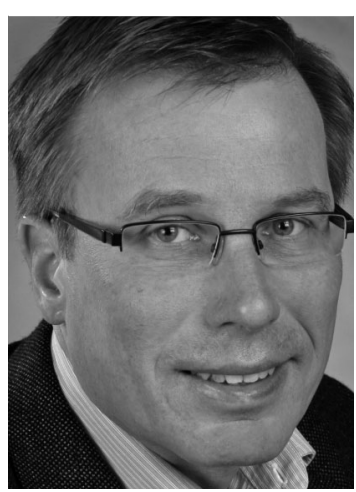

Prof. Dr. Dirk Richter

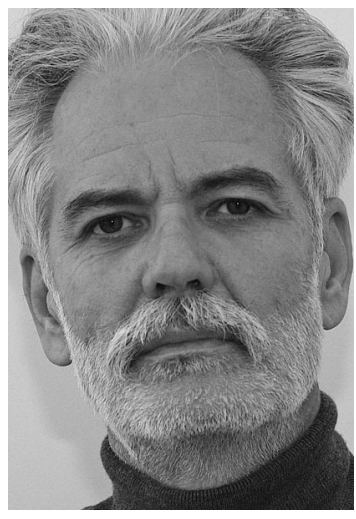

PD Dr. Holger Hoffmann

Bibliografie

Dol http://dx.doi.org/

10.1055/s-0029-1223380

Psychiat Prax 2009; 36:

357-358

(c) Georg Thieme Verlag KG

Stuttgart · New York.

ISSN 0303-4259

Korrespondenzadresse

Prof. Dr. Dirk Richter

Berner Fachhochschule

Fachbereich Gesundheit

Murtenstraße 10

3008 Bern, Schweiz

dirk.richter@bfh.ch
Die Situation gibt keinen Anlass zu Häme oder Selbstgerechtigkeit. Ein Durchbruch in der pharmakologischen Therapie schwerer psychischer Störungen wäre ein erheblicher, wenn nicht sogar ein entscheidender Schritt für die Psychiatrie als Fach und für die Akzeptanz bei Nutzerinnen und Nutzern, aber auch in der Bevölkerung gewesen. Leider stellt sich die Lage sowohl bezüglich der zweiten Generation der Antipsychotika als auch hinsichtlich der Antidepressiva heute anders dar. Hinter dem in wissenschaftlichen Zeitschriften publizierten Erfolg der neuen Generation antidepressiver Substanzen (i.d.R. SSRIs) stand offenbar eine selektive Publikationspraxis wie in zwei neueren Analysen mit Daten der amerikanischen Food and Drug Administration gefunden wurde $[1,2]$. Verständlicherweise verursachten diese Befunde eine erhebliche Diskussion auch in der deutschsprachigen Psychiatrie.

Nicht minder diskutiert wurden die Resultate von pharmaunabhängigen Studien für die Schizophreniebehandlung. Hinter den Akronymen CATIE, CUtLASS und EUFAST stehen Studien, welche die frühere Euphorie in der Schizophrenietherapie erheblich senkten $[3,4]$. Weder in der Effektivität noch insbesondere in der Akzeptanz durch die Patientinnen und Patienten war die zweite Generation der Antipsychotika der ersten überlegen, allenfalls das vermutlich geringere extrapyramidale Nebenwirkungsrisiko spricht für die neueren Neuroleptika.

In einer unlängst veröffentlichten Zusammenschau dieser Analysen vor allem unter dem Gesichtspunkt der Kosteneffektivität kamen Leucht und Kollegen zu der Einschätzung, dass die deutlich niedrigeren Kosten der ersten Generation der Antipsychotika hier einen praktischen und ökonomischen Vorteil versprechen würden [4]. In diesem Zusammenhang äußerten sie ein gewisses Verständnis für jetzt aufkommende Forderungen, die zur Verfügung stehenden finanziellen
Ressourcen für psychosoziale Interventionen anstatt der neueren Antipsychotika zu verwenden. Die Deutsche Gesellschaft für soziale Psychiatrie etwa fordert in einem aktuellen Memorandum eine Umkehr der Prioritäten, indem die pharmakologischen Interventionen nicht mehr von psychosozialen Interventionen begleitet werden, sondern letztere führend in der Therapie sein sollten [5]. Dieser Anspruch ist sicherlich sehr hoch, und es besteht die Gefahr, Erwartungen zu wecken, die momentan theoretisch wie empirisch kaum einlösbar sind. Sowohl die Soziologie als auch die Psychologie sind mit ähnlich hohen Ansprüchen in früheren Jahren und Jahrzehnten gescheitert [6,7].

Die Frage der Vorrangigkeit ist für uns momentan nachrangig, diese Frage kann letztlich nur empirisch beantwortet werden. Um allerdings zu einer verbesserten empirisch-basierten Einschätzung zu kommen, sehen wir in der aktuellen Situation eine Chance, die in den letzten Jahrzehnten vernachlässigten psychosozialen Interventionen in der Psychiatrie zum einen verstärkt zu erforschen und zum anderen die vorliegenden internationalen Forschungsresultate in diesem Therapiesektor auch tatsächlich in die Praxis umzusetzen.

Natürlich bilden die deutschsprachigen Länder keine Ausnahme hinsichtlich des eklatanten Mangels bei der Implementierung psychosozialer Therapie- und Betreuungsansätze. Es fällt allerdings schon auf, dass die Veröffentlichungen und Kongressbeiträge - beispielsweise bezüglich des Supported Employment oder Recovery - in einem erheblichen Missverhältnis zu den tatsächlich in die Praxis umgesetzten Programmen stehen. Diesbezüglich sind die angelsächsischen Länder schon etwas weiter, wenngleich die genannten Programme in der vermuteten Breite auch dort noch nicht zu finden sind.

Hinter dem skizzierten Mangel in der Umsetzung dieser Programme kann auf keinen Fall die feh- 
lende empirische Evidenz stehen. Wenngleich die psychosozialen Interventionen sicherlich nicht mit dem gleichen finanziellen Aufwand erforscht wurden wie die pharmakologischen Präparate, kann sich der Stand der Forschung durchaus sehen lassen:

- systematische Übersichten über die Effekte von Soteria-Projekten oder ähnlichen Einrichtungen haben eine geringere Medikamentendosis, eine höhere Patientenzufriedenheit bei ähnlichen Ergebnismessungen festgestellt [8,9],

- Recovery-Ansätze sind bis heute vielfach evaluiert worden; wenngleich die Outcome-Maße sehr uneinheitlich sind, konnte insbesondere bei kognitiven und sozialen Indikatoren für viele Nutzer eine Verbesserung festgestellt werden [10],

- der Erfolg von Supported-Employment-Programmen konnte mittlerweile auch in Europa nachgewiesen werden [11].

Zweifelsohne bedarf es weiterer und sicherlich auch verbesserter Forschungsanstrengungen. Bei der Rezeption und Implementierung der Forschungsergebnisse in die Praxis sehen wir im deutschsprachigen Raum jedoch die größten Defizite. Zu diesem Zweck schlagen wir vor, die überwiegend aus den angelsächsischen Ländern stammenden Ansätze nicht nur sprachlich auf die hiesigen Verhältnisse anzupassen, sondern auch inhaltlich an die hier anzutreffenden Versorgungsformen zu adaptieren. Für viele psychosoziale Programme existieren mittlerweile englischsprachiges didaktisches Material wie Manuale und Workbooks, welche auch evaluiert worden sind [15].

Ein gemeinsames Merkmal der Soteria-, Recovery- oder Supported-Employment-Ansätze ist der besondere Stellenwert, der eine deutlich andere Atmosphäre schaffenden Beziehung zwischen Professionellen und Nutzerinnen und Nutzern. Eine gute therapeutische Beziehung wird nicht nur von Nutzern immer wieder eingefordert und von Professionellen als bereichernd erlebt [12], sondern hat sich als eigenständige, wenngleich unspezifische Qualitätskomponente in vielen empirischen Untersuchungen herausgestellt [13]. Dies gilt es konzeptionell auszubauen und mit klaren Zielen in der Kommunikation zwischen Nutzer und Professionellen anzureichern.

Die bereits erfolgte Adaption des aus der Suchttherapie bekannten und bestens evaluierten Motivational-Interviewing-Ansatzes für die Allgemeine Psychiatrie könnte ein mögliches inhaltliches, aber auch didaktisches Verfahren sein, das für Mitarbeiterinnen und Mitarbeiter hierzulande attraktiv wäre [14]. Motivationslosigkeit ist bekanntlich eines der grundlegenden Probleme vieler chronisch psychisch kranker Menschen.

Angesichts des teils schon bestehenden, teils drohenden Mangels ärztlicher Mitarbeiter müssen die nichtärztlichen Berufsgruppen fachlich und organisatorisch deutlich aufgewertet werden, dies gilt vor allem für die psychiatrische Pflege. Eine Stärkung der psychosozialen Interventionen in der Versorgungspsychiatrie könnte den Stellenwert dieser Berufsgruppe erheblich verbessern. Ein weiteres Arbeitsfeld ist der die psychiatrischen Kliniken in der Langzeitversorgung zunehmend ablösende Wohn- und Heimbereich, der ebenfalls von zielgerichteten Interventionen auf der Basis verbesserter kommunikativer Beziehungen profitieren dürfte [16].

Im DGPPN-Auftrag wird zurzeit eine S3-Leitlinie für psychosoziale Therapien erstellt. Dieser Nachfolger der früheren Behandlungsleitlinie [17] ist hierzu ein wertvoller, aber sicher nicht aus- reichender Schritt. Wie in nahezu allen Bereichen der Medizin bieten Leitlinien auch in der Psychiatrie nicht die Gewähr für eine Implementierung. Der aktuellen Literatur zur Umsetzung der evidenzbasierter Erkenntnisse zufolge braucht es hier stärkere Irritationen, unter Umständen auch eine Re-Organisation [18]. Unter dem steigenden Kostendruck mit konsekutiver Verkürzung der stationären Aufenthaltsdauer und zunehmender Fragmentierung von Therapie und Rehabilitation dürfen wir nicht dem Irrtum verfallen, allein durch die Gabe neuer und teurer Medikamente könnten die Behandlungsverläufe nachhaltig verbessert werden. Für die Wirksamkeit der von uns genannten psychosozialen Interventionen gibt es hinreichende Evidenz, ihre KostenNutzen-Relation dürfte vergleichsweise besser sein und - für uns besonders wichtig - diese Interventionen verändern unseren $\mathrm{Zu}$ gang und die zukünftige Beziehungsgestaltung zu den Patienten. Lassen wir uns also irritieren.

\section{Literatur}

1 Kirsch I, Deacon BJ, Huedo-Medina T et al. Initial severity and antidepressant benefits: A meta-analysis of data submitted to the Food and Drug Administration. PLoS Med 2009; 5: e45

2 Turner EH, Matthews AM, Linardatos E et al. Selective publication of antidepressant trials and its influence on apparent efficacy. N Engl J Med 2009; 358: 252-260

3 Lewis S, Lieberman J. CATIE and CUtLASS: Can we handle the truth? $\mathrm{Br} J$ Psychiatry 2008; 192: 161-163

4 Leucht S, Kissling W, Davis JM. Second-generation antipsychotics for schizophrenia: Can we solve the conflict? Psychol Med 2009; 39: 1591-1602

5 DGSP. Memorandum der Deutschen Gesellschaft für Soziale Psychiatrie e.V. - DGSP - zur Verwendung von Antipsychotika. Köln: 2009

6 Bentall $R$. Doctoring the mind: Why psychiatric treatments fail. London: Allen Lane, 2009

7 Richter D. Psychisches System und soziale Umwelt: Soziologie psychischer Störungen in der Ära der Biowissenschaften. Bonn: Psychiatrie-Verlag, 2003

8 Calton T, Ferriter M, Huband $N$ et al. A systematic review of the Soteria paradigm for the treatment of people diagnosed with schizophrenia. Schizophr Bull 2008; 34: 181-192

9 Lloyd-Lewis B, Slade M, Jagielska D et al. Residential alternatives to acute psychiatric hospital admission: Systematic review. Br J Psychiatry 2009; 195: 109-117

10 Lieberman JA, Drake RE, Sederer LI et al. Science and recovery in schizophrenia. Psychiatr Serv 2008; 59: 487-496

11 Burns T, Catty J, Becker T et al, for the EQOLISE Group. The effectiveness of supported employment for people with severe mental illness: a randomised controlled trial. Lancet 2007; 370: 1146-1152

12 Richter D, Hahn S. Formelles und informelles Aufgabenprofil in der ambulanten psychiatrischen Pflege aus Sicht von Pflegenden: Eine MetaSynthese. Pflege 2009; 22: 129-142

13 Priebe S, McCabe $R$. The therapeutic relationship in psychiatric settings. Acta Psychiatr Scand 2006; 113 (Suppl. 429): 69-72

14 Arkowitz H, Westra HA, Miller WR (eds). Motivational Interviewing in the Treatment of Psychological Problems. London: Guilford, 2007

15 Barbic S, Krupa T, Armstrong I. A randomized controlled trial of the effectiveness of a modified recovery workbook program: Preliminary findings. Psychiatr Serv 2009; 60: 491-497

16 Bitter D, Entenfellner A, Matschnig T et al. Da-Heim im Heim? Bedeutet Ent-Hospitalisierung auch Ent-Deinstituationalisierung? Psychiat Prax 2009; 36: 261-269

17 Becker T, Reker T, Weig W (eds). Behandlungsleitlinie Psychosoziale Therapien. Darmstadt: Steinkopff, 2005

18 Drake RE, Bond GR, Essock SM. Implementing evidence-based practices for people with schizophrenia. Schizophr Bull 2009 Online Access: doi $10.1093 / \mathrm{schbul} / \mathrm{spb} 041$ (04.10.2009) 